\title{
ON UNCOUNTABLE $K$-BESSEL AND $K$-HILBERT SYSTEMS IN NONSEPARABLE BANACH SPACES
}

\author{
MIGDAD I. ISMAILOV
}

\begin{abstract}
The concepts of the uncountable $K$-Bessel and $K$-Hilbert systems in nonseparable Banach spaces are introduced in this work. The definition for uncountable unconditional basis is given and the criterion for uncountable unconditional basicity is found. Banach space of systems of scalars $K$ is considered and the concepts of $K$-Bessel and $K$-Hilbert systems with respect to this space are introduced in nonseparable Banach spaces. $K$-Besselianness and $K$-Hilbertianness criteria for a system are found. $K$-Besselianness and $K$-Hilbertianness of a system are studied in case where the space $K$ is generated by an uncountable unconditional basis. Non-separable Banach spaces $L_{p}^{V}(R)$ and $l_{p}(R)(1 \leq p<+\infty)$ are constructed. It is shown that in case $p \geq 2$ the system $\left\{e^{i \alpha t}\right\}_{\alpha \in R}$ is $l_{p}(R)$-Besselian, and in case $p \leq 2$ it is $l_{p}(R)$-Hilbertian in $L_{p}^{V}(R)$.
\end{abstract}

\section{Introduction}

The concept of frame has been probably introduced by R.J. Duffin and A.C. Schaeffer in 1952 in [17] the study of non-harmonic Fourier series with respect to perturbed exponential systems. In this seminal work, Duffin and Schaefer established some properties of exponential frames. In the same work, they introduced the concept of abstract frame in separable Hilbert space and extended some properties of frames consisting of perturbed exponential systems to this concept. The interest to frames has grown in the 1980s due to wide applications of wavelet methods in various fields of natural science. Standing at the crossroads of theory and practice, the wavelets are widely used in processing and encoding of signals and different kinds of images (satellite images, roentgenographies of internal organs, etc), in pattern recognition, in the study of the properties of crystal surfaces and nano-objects, and in many other fields. For theoretical aspects of this direction we refer the readers to Ch. Chui [13], Y. Meyer [25], I. Daubechies [18], S. Mallat [25], R. Young [34], Ch. Heil [22], O. Christensen [14]-[16], etc.

In subsequent years, the concept of frame has been generalized to various mathematical structures (for example, Banach frames, $p$-frames, etc) and new methods for establishing frames have been elaborated. One of these methods is a perturbation method. A lot of results have been obtained in this direction in

2010 Mathematics Subject Classification. 41A10, 41A28, 42A10.

Key words and phrases. nonseparable Banach space, uncountable unconditional basis, $K$ Besselianness and $K$-Hilbertianness. 
the context of classical Paley-Wiener theorem on perturbation of an orthonormal basis (for more details see O. Christensen [14]-[16] and Ch. Heil [22]).

Frames in Banach spaces were first considered by K.H. Gröchenig [21] in 1991. He introduced the concepts of atomic decomposition and Banach frame. It should be noted that, unlike Hilbert's case, the definition of Banach frame does not, in general, provide the decomposition of arbitrary element of Banach space (or of arbitrary element of the closure of the linear span of the system under consideration). In special cases, such a decomposition exists. $L^{p}$-case has been considered by A. Aldroubi, Q. Sun, W.-Sh. Tang in [1] where the concept of $p$-frame has been introduced and the atomic decomposition with regard to $L_{p}$-subspaces invariant with respect to the shift operator has been obtained. This idea has been extended to the general Banach case by Christensen O. and Stoeva D. T. [16]. Also, the concept of $q$-Riesz basis for a Banach space has been introduced in these works, which generalizes the one of Riesz basis introduced by N.K. Bari [13]. Note that similar results have been obtained in [3]-[5]. There are different generalizations of frames, and this research field has been continuously growing over the last years (see, e.g., [1], [7]-[10], [16], [19], [20], [23], [26]-[28]).

Note that, in general, the case of non-separable space is not considered in the approximation theory due to objective reasons. The examples with non-separable spaces are mostly exotic. Meanwhile, from a purely theoretical point of view, it would be interesting to develop approximation theory to the case of non-separable space. But, to do so, of course you first have to define the corresponding concepts of the theory of Bessel-Hilbert systems and the theory of frames for the case of non-separable space, and then to extend the basic facts of these theories to nonseparable case. Perhaps, this was first done in [11], [12], where the concept of uncountable Hilbert frame was defined and the basic provisions of the above theories were extended to non-separable case.

In this work, we study the Besselianness and Hilbertianness of a minimal system in non-separable Banach spaces. The concept of uncountable unconditional basis and those of $K$-Bessel and $K$-Hilbert systems with respect to nonseparable Banach space of systems of scalars $K$ are introduced. Criteria of uncountable unconditional basicity, $K$-Besselianness and $K$-Hilbertianness for systems are found, and the relationship between them is studied. Examples are given.

\section{Uncountable Unconditional Basis In Nonseparable Banach Spaces}

Let $X$ be a nonseparable Banach space with the norm $\|\cdot\|_{X}, I$ be an uncountable index set, $I^{a}$ be a set of no-more-than countable subsets of $I, I_{0}$ be a set of finite subsets of $I$, and $\left\{\varphi_{\alpha}\right\}_{\alpha \in I}$ be some system in $X$.

Definition 2.1. The system $\left\{\varphi_{\alpha}\right\}_{\alpha \in I}$ is called an uncountable unconditional basis in $X$ if

$\forall x \in X \exists ! \lambda=\left\{\lambda_{\alpha}\right\}_{\alpha \in I}:\left\{\alpha \in I: \lambda_{\alpha} \neq 0\right\} \in I^{a} x=\sum_{\alpha \in I} \lambda_{\alpha} \varphi_{\alpha}$ (unconditionally).

Example 2.1. Let $l_{p}(I), 1 \leq p<+\infty$, be a set of systems of scalars $\lambda=\left\{\lambda_{\alpha}\right\}_{\alpha \in I}$ such that $\omega_{\lambda}=\left\{\alpha \in I: \lambda_{\alpha} \neq 0\right\} \in I^{a}$ and $\sum_{\alpha \in \omega_{\lambda}}\left|\lambda_{\alpha}\right|^{p}<+\infty . l_{p}(I)$ is a Banach 
space with the norm

$$
\|\lambda\|=\left(\sum_{\alpha \in \omega_{\lambda}}\left|\lambda_{\alpha}\right|^{p}\right)^{\frac{1}{p}}, \lambda=\left\{\lambda_{\alpha}\right\}_{\alpha \in I} \in l_{p}(I) .
$$

Let $\delta_{\alpha \beta}$ be the Kronecker symbol and $\delta_{\alpha}=\left\{\delta_{\alpha \beta}\right\}_{\beta \in I}$. The system $\left\{\delta_{\alpha}\right\}_{\alpha \in I}$ is an uncountable unconditional basis for $l_{p}(I)$. In fact, it is not difficult to show that for $\forall \lambda=\left\{\lambda_{\alpha}\right\}_{\alpha \in I} \in l_{p}(I)$ there exists a unique representation in the form of unconditionally convergent series $\lambda=\sum_{\alpha \in \omega_{\lambda}} \lambda_{\alpha} \delta_{\alpha}$.

Let's state a criterion for uncountable unconditional basicity in nonseparable Banach spaces.

Theorem 2.1. In order for the system $\left\{\varphi_{\alpha}\right\}_{\alpha \in I}$ to be an uncountable unconditional basis in $X$, it is necessary and sufficient that the following conditions hold:

1) the system $\left\{\varphi_{\alpha}\right\}_{\alpha \in I}$ is complete in $X$;

2) the system $\left\{\varphi_{\alpha}\right\}_{\alpha \in I}$ is minimal in $X$;

$$
3) \exists M>0: \forall J \in I_{0}, \forall x \in X\left\|\sum_{\alpha \in J} \varphi_{\alpha}^{*}(x) \varphi_{\alpha}\right\|_{X} \leq M\|x\|_{X},
$$

where $\left\{\varphi_{\alpha}^{*}\right\}_{\alpha \in I}$ is a system biorthogonal to $\left\{\varphi_{\alpha}\right\}_{\alpha \in I}$.

Proof. Necessity. Let $\left\{\varphi_{\alpha}\right\}_{\alpha \in I}$ form an uncountable unconditional basis for $X$. Then it is obvious that $\left\{\varphi_{\alpha}\right\}_{\alpha \in I}$ is complete in $X$. Denote by $K_{\varphi}$ the set of systems of scalars $\lambda=\left\{\lambda_{\alpha}\right\}_{\alpha \in I}$ such that $\left\{\alpha \in I: \lambda_{\alpha} \neq 0\right\} \in I^{a}$ and the series $\sum_{\alpha \in I} \lambda_{\alpha} \varphi_{\alpha}$ is unconditionally convergent. It is clear that $K_{\varphi}$ becomes a linear space with linear operations

$$
\begin{gathered}
\forall \lambda=\left\{\lambda_{\alpha}\right\}_{\alpha \in I}, \mu=\left\{\mu_{\alpha}\right\}_{\alpha \in I} \in K_{\varphi}, \forall k \\
\lambda+\mu=\left\{\lambda_{\alpha}+\mu_{\alpha}\right\}_{\alpha \in I}, k \lambda=\left\{k \lambda_{\alpha}\right\}_{\alpha \in I} .
\end{gathered}
$$

Let's show that $K_{\varphi}$ is a Banach space with the norm

$$
\|\lambda\|_{K_{\varphi}}=\sup _{J \in I_{0}}\left\|\sum_{\alpha \in J} \lambda_{\alpha} \varphi_{\alpha}\right\|_{X}, \lambda=\left\{\lambda_{\alpha}\right\}_{\alpha \in I} \in K .
$$

Let's first verify the axioms of norm. 1) It is clear that $\|\lambda\|_{K_{\varphi}} \geq 0$, and if

$$
\|\lambda\|_{K_{\varphi}}=\sup _{J \in I_{0}}\left\|\sum_{\alpha \in J} \lambda_{\alpha} \varphi_{\alpha}\right\|_{X}=0
$$

then $\forall \alpha \in I\left\|\lambda_{\alpha} \varphi_{\alpha}\right\|_{X}=0$. Consequently, $\lambda_{\alpha}=0$, i.e. $\left.\lambda=0.2\right) \forall k$ we have

$$
\|k \lambda\|_{K_{\varphi}}=\sup _{J \in I_{0}}\left\|\sum_{\alpha \in J} k \lambda_{\alpha} \varphi_{\alpha}\right\|_{X}=|k| \sup _{J \in I_{0}}\left\|\sum_{\alpha \in J} \lambda_{\alpha} \varphi_{\alpha}\right\|_{X}=|k|\|\lambda\|_{K_{\varphi}} .
$$

3) Let $\forall \lambda=\left\{\lambda_{\alpha}\right\}_{\alpha \in I}, \mu=\left\{\mu_{\alpha}\right\}_{\alpha \in I} \in K_{\varphi}$. For $\forall J^{\prime} \in I_{0}$ we have

$$
\left\|\sum_{\alpha \in J^{\prime}}\left(\lambda_{\alpha}+\mu_{\alpha}\right) \varphi_{\alpha}\right\|_{X} \leq\left\|\sum_{\alpha \in J^{\prime}} \lambda_{\alpha} \varphi_{\alpha}\right\|_{X}+\left\|\sum_{\alpha \in J^{\prime}} \mu_{\alpha} \varphi_{\alpha}\right\|_{X} \leq
$$




$$
\leq \sup _{J \in I_{0}}\left\|\sum_{\alpha \in J} \lambda_{\alpha} \varphi_{\alpha}\right\|_{X}+\sup _{J \in I_{0}}\left\|\sum_{\alpha \in J} \mu_{\alpha} \varphi_{\alpha}\right\|_{X}=\|\lambda\|_{K_{\varphi}}+\|\mu\|_{K_{\varphi}} .
$$

Hence

$$
\|\lambda+\mu\|_{K_{\varphi}} \leq\|\lambda\|_{K_{\varphi}}+\|\mu\|_{K_{\varphi}} .
$$

Let's prove the completeness of the space $K_{\varphi}$. Let $\lambda_{n}=\left\{\lambda_{\alpha}^{(n)}\right\}_{\alpha \in I}, n \in N$, be some fundamental sequence in $K_{\varphi}$, i.e. the following condition hold:

$$
\forall \varepsilon>0 \exists n_{0}(\varepsilon) \forall n, m \geq n_{0}(\varepsilon)\left\|\lambda_{n}-\lambda_{m}\right\|_{K_{\varphi}}=\sup _{J \in I_{0}}\left\|\sum_{\alpha \in J}\left(\lambda_{\alpha}^{(n)}-\lambda_{\alpha}^{(m)}\right) \varphi_{\alpha}\right\|_{X}<\varepsilon .
$$

From (2.1) it follows that

$$
\forall J \in I_{0}\left\|\sum_{\alpha \in J}\left(\lambda_{\alpha}^{(n)}-\lambda_{\alpha}^{(m)}\right) \varphi_{\alpha}\right\|_{X}<\varepsilon .
$$

In particular, $\forall \alpha \in I\left|\lambda_{\alpha}^{(n)}-\lambda_{\alpha}^{(m)}\right|<\frac{\varepsilon}{\left\|\varphi_{\alpha}\right\|_{X}}$. Therefore, $\forall \alpha \in I$ the sequence $\left\{\lambda_{\alpha}^{(n)}\right\}_{n \in N}$ is fundamental, and hence, it is convergent. Let $\lambda_{\alpha}=\lim _{n \rightarrow \infty} \lambda_{\alpha}^{(n)}$. Assume $A_{n}=\left\{\alpha \in I: \lambda_{\alpha}^{(n)} \neq 0\right\}$ and $A=\bigcup_{n=1}^{\infty} A_{n}$. Obviously, $A \in I^{a}$. On the other hand, from $I \backslash A=\bigcap_{n=1}^{\infty}\left(I \backslash A_{n}\right)$ we obtain that $I \backslash A \subset\left\{\alpha \in I: \lambda_{\alpha}=0\right\}$, i.e. $\left\{\alpha \in I: \lambda_{\alpha} \neq 0\right\} \subset A$. Therefore, $\left\{\alpha \in I: \lambda_{\alpha} \neq 0\right\} \in I^{a}$. Passing to the limit in $(2.2)$ as $m \rightarrow \infty$, we obtain

$$
\forall \varepsilon>0 \exists n_{0}(\varepsilon) \forall n \geq n_{0}(\varepsilon)\left\|\sum_{\alpha \in J}\left(\lambda_{\alpha}^{(n)}-\lambda_{\alpha}\right) \varphi_{\alpha}\right\|_{X} \leq \varepsilon, \forall J \in I_{0} .
$$

Let $\left\{\lambda_{\alpha_{i}}\right\}_{i \in N}$ be an arbitrary transposition of elements of the system $\lambda=\left\{\lambda_{\alpha}\right\}_{\alpha \in I}$. Let's show the convergence of the series $\sum_{i=1}^{\infty} \lambda_{\alpha_{i}} \varphi_{\alpha_{i}}$. Consider $\forall \varepsilon>0$ and $\forall k, p \in N$. According to $(2.3), \forall n \geq n_{0}(\varepsilon)$ :

$$
\left\|\sum_{i=1}^{k}\left(\lambda_{\alpha_{i}}^{(n)}-\lambda_{\alpha_{i}}\right) \varphi_{\alpha_{i}}\right\|_{X} \leq \frac{\varepsilon}{4} \text { and }\left\|\sum_{i=1}^{k+p}\left(\lambda_{\alpha_{i}}^{(n)}-\lambda_{\alpha_{i}}\right) \varphi_{\alpha_{i}}\right\|_{X} \leq \frac{\varepsilon}{4}
$$

Let $n \geq n_{0}(\varepsilon)$ be a fixed number. From the convergence of the series $\sum_{i=1}^{\infty} \lambda_{\alpha_{i}}^{(n)} \varphi_{\alpha_{i}}$ we obtain that $\exists k_{0}(\varepsilon) \forall k \geq k_{0}(\varepsilon)$ the inequality

$$
\left\|\sum_{i=1}^{k} \lambda_{\alpha_{i}}^{(n)} \varphi_{\alpha_{i}}-\sum_{i=1}^{k+p} \lambda_{\alpha_{i}}^{(n)} \varphi_{\alpha_{i}}\right\|_{X}<\frac{\varepsilon}{2}
$$

holds. Then, in view of (2.4) and (2.5), we obtain

$$
\left\|\sum_{i=1}^{k} \lambda_{\alpha_{i}} \varphi_{\alpha_{i}}-\sum_{i=1}^{k+p} \lambda_{\alpha_{i}} \varphi_{\alpha_{i}}\right\|_{X} \leq
$$




$$
\begin{gathered}
\leq\left\|\sum_{i=1}^{k} \lambda_{\alpha_{i}} \varphi_{\alpha_{i}}-\sum_{i=1}^{k} \lambda_{\alpha_{i}}^{(n)} \varphi_{\alpha_{i}}\right\|_{X}+ \\
+\left\|\sum_{i=1}^{k} \lambda_{\alpha_{i}}^{(n)} \varphi_{\alpha_{i}}-\sum_{i=1}^{k+p} \lambda_{\alpha_{i}}^{(n)} \varphi_{\alpha_{i}}\right\|_{X}+\left\|\sum_{i=1}^{k+p} \lambda_{\alpha_{i}} \varphi_{\alpha_{i}}-\sum_{i=1}^{k+p} \lambda_{\alpha_{i}}^{(n)} \varphi_{\alpha_{i}}\right\|_{X}<\varepsilon,
\end{gathered}
$$

and therefore, the series $\sum_{i=1}^{\infty} \lambda_{\alpha_{i}} \varphi_{\alpha_{i}}$ is convergent. Thus, the series $\sum_{\alpha \in I} \lambda_{\alpha} \varphi_{\alpha}$ is unconditionally convergent. Further, by virtue of (2.3) we have

$$
\forall \varepsilon>0 \exists n_{0}(\varepsilon) \forall n \geq n_{0}(\varepsilon)\left\|\lambda_{n}-\lambda\right\|_{K_{\varphi}}=\sup _{J \in I_{0}}\left\|\sum_{\alpha \in J}\left(\lambda_{\alpha}^{(n)}-\lambda_{\alpha}\right) \varphi_{\alpha}\right\|_{X} \leq \varepsilon,
$$

i.e. $\lambda_{n}$ converge in the space $K_{\varphi}$ to the element $\lambda$. Therefore, the space $K_{\varphi}$ is complete. Consider the operator $F: K_{\varphi} \rightarrow X$ defined by the formula $F \lambda=x$, $\lambda=\left\{\lambda_{\alpha}\right\}_{\alpha \in I}$, where $x=\sum_{\alpha \in I} \lambda_{\alpha} \varphi_{\alpha} . F$ is a linear bounded operator. In fact, the linearity of $F$ is obvious, and its boundedness follows from the relation

$$
\|F \lambda\|_{X}=\left\|\sum_{\alpha \in I} \lambda_{\alpha} \varphi_{\alpha}\right\|_{X} \leq \sup _{J \in I_{0}}\left\|\sum_{\alpha \in J} \lambda_{\alpha} \varphi_{\alpha}\right\|_{X}=\|\lambda\|_{K_{\varphi}} .
$$

Further, as the operator $F$ maps $K_{\varphi}$ one-to-one onto $X$, the Banach theorem implies that the operator $F^{-1}$ is bounded.

Now let's prove the minimality of the system $\left\{\varphi_{\alpha}\right\}_{\alpha \in I}$. Define the functional $\varphi_{\alpha}^{*}(x)=\lambda_{\alpha}, \forall \alpha \in I$, where $\lambda=F^{-1} x$. It is easily seen that such a functional is linear. Let's show it is bounded. We have

$$
\begin{gathered}
\left|\varphi_{\alpha}^{*}(x)\right|=\left|\lambda_{\alpha}\right|=\frac{\left\|\lambda_{\alpha} \varphi_{\alpha}\right\|_{X}}{\left\|\varphi_{\alpha}\right\|_{X}} \leq \frac{1}{\left\|\varphi_{\alpha}\right\|_{X}} \sup _{J \in I_{0}}\left\|\sum_{\beta \in J} \lambda_{\beta} \varphi_{\beta}\right\|_{X} \\
=\frac{1}{\left\|\varphi_{\alpha}\right\|}\|\lambda\|_{K_{\varphi}} \leq \frac{\left\|F^{-1}\right\|}{\left\|\varphi_{\alpha}\right\|_{X}}\|x\|_{X},
\end{gathered}
$$

i.e.

$$
\left\|\varphi_{\alpha}^{*}\right\| \leq \frac{\left\|F^{-1}\right\|}{\left\|\varphi_{\alpha}\right\|_{X}}
$$

On the other hand, $\varphi_{\alpha}^{*}\left(\varphi_{\beta}\right)=\delta_{\alpha \beta}$, where $\delta_{\alpha \beta}$ is the Kronecker symbol. Thus, the systems $\left\{\varphi_{\alpha}\right\}_{\alpha \in I}$ and $\left\{\varphi_{\alpha}^{*}\right\}_{\alpha \in I}$ are biorthogonal.

Finally, $\forall x \in X$ and $\forall J^{\prime} \in I_{0}$ we have

$$
\left\|\sum_{\alpha \in J^{\prime}} \varphi_{\alpha}^{*}(x) \varphi_{\alpha}\right\|_{X} \leq \sup _{J \in I_{0}}\left\|\sum_{\alpha \in J} \varphi_{\alpha}^{*}(x) \varphi_{\alpha}\right\|=\left\|F^{-1} x\right\| \leq\left\|F^{-1}\right\|\|x\|_{X},
$$

i.e. the condition 3 ) holds.

Sufficiency. Let the conditions 1) - 3) hold. Consider $\forall x \in X$. The completeness of the system $\left\{\varphi_{\alpha}\right\}_{\alpha \in I}$ in $X$ implies

$$
\forall \varepsilon>0 \exists y \in L\left(\left\{\varphi_{\alpha}\right\}_{\alpha \in I}\right):\|x-y\|_{X}<\varepsilon .
$$

Then

$$
\forall n \in N \exists y_{n} \in L\left(\left\{\varphi_{\alpha}\right\}_{\alpha \in I}\right):\left\|x-y_{n}\right\|_{X}<\frac{1}{n}
$$


Let

$$
J_{n} \in I_{0}: y_{n}=\sum_{\alpha \in J_{n}} \lambda_{\alpha} \varphi_{\alpha}=\sum_{\alpha \in J_{n}} \varphi_{\alpha}^{*}\left(y_{n}\right) \varphi_{\alpha}
$$

Denote $\omega=\bigcup_{n=1}^{\infty} J_{n}$. Obviously, $\omega \in I^{a}$.

Take arbitrary $\varepsilon>0$. Let $n_{0} \in N: \frac{1}{n_{0}}<\varepsilon$. For $\forall J \subset \omega: J_{n_{0}} \subset J$ we have

$$
y_{n_{0}}=\sum_{\alpha \in J_{n_{0}}} \varphi_{\alpha}^{*}\left(y_{n}\right) \varphi_{\alpha}=\sum_{\alpha \in J} \varphi_{\alpha}^{*}\left(y_{n_{0}}\right) \varphi_{\alpha}
$$

Then, using (2.6), (2.7) and the condition 3), $\forall J \subset \omega: J_{n_{0}} \subset J$ we obtain

$$
\begin{gathered}
\left\|x-\sum_{\alpha \in J} \varphi_{\alpha}^{*}(x) \varphi_{\alpha}\right\|_{X}=\left\|x-y_{n_{0}}+y_{n_{0}}-\sum_{\alpha \in J} \varphi_{\alpha}^{*}(x) \varphi_{\alpha}\right\|_{X} \leq \\
\leq\left\|x-y_{n_{0}}\right\|_{X}+\left\|\sum_{\alpha \in J} \varphi_{\alpha}^{*}\left(x-y_{n_{0}}\right) \varphi_{\alpha}\right\|_{X} \leq\left\|x-y_{n_{0}}\right\|_{X}+M\left\|x-y_{n_{0}}\right\|_{X}= \\
=(1+M)\left\|x-y_{n_{0}}\right\|_{X}<(1+M) \varepsilon .
\end{gathered}
$$

Thus

$$
\forall \varepsilon>0 \exists J_{0} \subset \omega \forall J \subset \omega: J_{0} \subset J\left\|x-\sum_{\alpha \in J} \varphi_{\alpha}^{*}(x) \varphi_{\alpha}\right\|_{X}<\varepsilon,
$$

and therefore, due to the criterion for unconditional convergence of series, the series $\sum_{\alpha \in \omega} \varphi_{\alpha}^{*}(x) \varphi_{\alpha}$ converges unconditionally to $x \in X$. Obviously, due to the minimality of the system $\left\{\varphi_{\alpha}\right\}_{\alpha \in \omega}$, this representation is unique. i.e. the system $\left\{\varphi_{\alpha}\right\}_{\alpha \in \omega}$ forms an uncountable unconditional basis for $X$.

Corollary 2.1. Let $\left\{\varphi_{\alpha}\right\}_{\alpha \in I}$ be an uncountable unconditional basis in $X$ and $\left\{\varphi_{\alpha}^{*}\right\}_{\alpha \in I}$ be a system biorthogonal to $\left\{\varphi_{\alpha}\right\}_{\alpha \in I}$. Then $\left\{\varphi_{\alpha}^{*}\right\}_{\alpha \in I}$ forms an uncountable unconditional basis for $\overline{L\left(\left\{\varphi_{\alpha}^{*}\right\}_{\alpha \in I}\right)}$.

Proof. It suffices to prove the validity of the condition 3) of Theorem 2.1. For $\forall f \in X^{*}$ we have

$$
\left\|\sum_{\alpha \in J} f\left(\varphi_{\alpha}\right) \varphi_{\alpha}^{*}\right\|_{X^{*}}=\sup _{\|x\|=1}\left|\sum_{\alpha \in J} f\left(\varphi_{\alpha}\right) \varphi_{\alpha}^{*}(x)\right|=\sup _{\|x\|=1}\left|f\left(\sum_{\alpha \in J} \varphi_{\alpha}^{*}(x) \varphi_{\alpha}\right)\right| \leq M\|f\|_{X} .
$$

\section{3. $K$-Bessel and $K$-Hilbert Systems}

Let $X$ be a nonseparable Banach space, $I$ be some uncountable index set, and $I^{a}$ be a set of no-more-than countable subsets of $I$. Consider the minimal system $\left\{x_{\alpha}\right\}_{\alpha \in I} \subset X$ with the biorthogonal system $\left\{x_{\alpha}^{*}\right\}_{\alpha \in I} \subset X^{*}$. Let $K$ be a nonseparable Banach space of systems of scalars $\lambda=\left\{\lambda_{\alpha}\right\}_{\alpha \in I}$ such that $\left\{\alpha \in I: \lambda_{\alpha} \neq 0\right\} \in I^{a}$. 
The space $K$ is called a $C B$-space if the system $\left\{\delta_{\alpha}\right\}_{\alpha \in I} \subset K, \delta_{\alpha}=\left\{\delta_{\alpha \beta}\right\}_{\beta \in I}$ forms an uncountable unconditional basis for $K$, i.e. $\forall \lambda=\left\{\lambda_{\alpha}\right\}_{\alpha \in I} \in K$ the relation

$$
\lambda=\sum_{\alpha \in I} \lambda_{\alpha} \delta_{\alpha}=\sum_{i=1}^{\infty} \lambda_{\alpha_{i}} \delta_{\alpha_{i}}
$$

holds, where $\left\{\lambda_{\alpha_{i}}\right\}_{i \in N}$ is a sequence of arbitrary permutations of non-zero elements $\lambda=\left\{\lambda_{\alpha}\right\}_{\alpha \in I}$. Let $\left\{\delta_{\alpha}^{*}\right\}_{\alpha \in I} \subset K^{*}$ be a system biorthogonal to $\left\{\delta_{\alpha}\right\}_{\alpha \in I}$.

The next definition is a generalization of the concept of Bessel sequence.

Definition 3.1. The pair $\left\{x_{\alpha} ; x_{\alpha}^{*}\right\}$ is called $K$-Bessel if the condition $\left\{x_{\alpha}^{*}(x)\right\}_{\alpha \in I} \in$ $K, \forall x \in X$, holds. If the system $\left\{x_{\alpha}\right\}_{\alpha \in I}$ is complete in $X$ and the pair $\left\{x_{\alpha} ; x_{\alpha}^{*}\right\}$ is $K$-Bessel in $X$, then $\left\{x_{\alpha}\right\}_{\alpha \in I}$ is called $K$-Bessel in $X$.

Example 3.1. Let $e_{\alpha}(t)=e^{i \alpha t}, t \in R$. Assume $V=\operatorname{span}\left\{e_{\alpha}\right\}_{\alpha \in R}$. It is clear that $\forall x \in V \exists M_{x}:|x(t)| \leq M_{x}$. Therefore $\forall p \in[1 ;+\infty)$ there exists $\varlimsup_{T \rightarrow \infty} \frac{1}{2 T} \int_{-T}^{T}|x(t)|^{p} d t$. Let's show that

$$
\|x\|_{p}=\varlimsup_{T \rightarrow \infty}\left(\frac{1}{2 T} \int_{-T}^{T}|x(t)|^{p} d t\right)^{\frac{1}{p}}
$$

is a norm in $V$. Obviously, $\|x\|_{p} \geq 0$. Let $\|x\|_{p}=0$ and $x=\sum_{k} c_{k} e^{i \alpha_{k} t}$. For $\forall p \in[1 ;+\infty)$, from

$\frac{1}{2 T} \int_{-T}^{T}|x(t)|^{2} d t=\int_{-T}^{T}(2 T)^{-\frac{1}{p}}|x(t)|(2 T)^{-\frac{1}{q}}|x(t)| d t \leq M_{x}\left(\frac{1}{2 T} \int_{-T}^{T}|x(t)|^{p} d t\right)^{\frac{1}{p}}$

we obtain

$$
\varlimsup_{T \rightarrow \infty} \frac{1}{2 T} \int_{-T}^{T}|x(t)|^{2} d t \leq M_{x} \varlimsup_{T \rightarrow \infty}\left(\frac{1}{2 T} \int_{-T}^{T}|x(t)|^{p} d t\right)^{\frac{1}{p}}=0 .
$$

However

$$
\varlimsup_{T \rightarrow \infty} \frac{1}{2 T} \int_{-T}^{T}|x(t)|^{2} d t=\sum_{k}\left|c_{k}\right|^{2},
$$

hence, $x=0$. Further, $\forall \lambda$ we have

$$
\|\lambda x\|_{p}=\varlimsup_{T \rightarrow \infty}\left(\frac{1}{2 T} \int_{-T}^{T}|\lambda x(t)|^{p} d t\right)^{\frac{1}{p}}=|\lambda|\|x\|_{p} .
$$

At last, by Minkowski inequality,

$$
\left(\frac{1}{2 T} \int_{-T}^{T}|x(t)+y(t)|^{p} d t\right)^{\frac{1}{p}} \leq\left(\frac{1}{2 T} \int_{-T}^{T}|x(t)|^{p} d t\right)^{\frac{1}{p}}+\left(\frac{1}{2 T} \int_{-T}^{T}|x(t)|^{p} d t\right)^{\frac{1}{p}} .
$$

Passing to the upper limit as $T \rightarrow+\infty$ we obtain $\|x+y\|_{p} \leq\|x\|_{p}+\|y\|_{p}$. Consequently, $\|\cdot\|_{p}$ is a norm in the linear space $V$. Denote the obtained normed space by $V_{p}$. Define a scalar product in the space $V_{2}$ as follows:

$$
(x, y)_{V}=\lim _{T \rightarrow \infty} \frac{1}{2 T} \int_{-T}^{T} x(t) \overline{y(t)} d t .
$$


The system $\left\{e_{\alpha}\right\}_{\alpha \in R}$ is orthonormal in $V_{2}$. Denote by $L_{p}^{V}(R), 1 \leq p<+\infty$, the completion of the space $V_{p}$. Using Holder inequality, it is not difficult to show that $L_{p}^{V}(R) \subset L_{2}^{V}(R)$ for $p>2$ and $L_{2}^{V}(R) \subset L_{p}^{V}(R)$ for $p<2$. The space $L_{p}^{V}(R)$ is non-separable. To show this, it obviously suffices to show the non-separability of the space $V_{p}$.

Note that if there exist an uncountable set $M$ and a number $\varepsilon_{0}>0$ in the metric space $(E, \rho)$ such that for any two different $x, y \in M$ the inequality $\rho(x, y) \geq \varepsilon_{0}$ holds, then $(E, \rho)$ is non-separable. In fact, let $(E, \rho)$ be separable and $\left\{e_{n}\right\}_{n \in N}$ be dense in $(E, \rho)$. Consider a countable system of balls $S_{n}=S\left(e_{n}, \frac{\varepsilon_{0}}{3}\right)$. Then, in some ball $S_{n}$ there exist more than one point of $M$. Let $x, y \in M \cap S_{n}$. Then

$$
\varepsilon_{0} \leq \rho(x, y) \leq \rho\left(x, e_{n}\right)+\rho\left(y, e_{n}\right)<\frac{\varepsilon_{0}}{3}+\frac{\varepsilon_{0}}{3}=\frac{2 \varepsilon_{0}}{3} .
$$

But this is a contradiction. So $(E, \rho)$ is non-separable.

Let's first establish the non-separability of the space $V_{1}$. Let $M=\left\{e_{\alpha}\right\}_{\alpha \in R}$. Let's estimate the number $\left\|e_{\alpha}-e_{\beta}\right\|_{1}$ for different $\forall e_{\alpha}, e_{\beta} \in M$. As the system $\left\{e_{\alpha}\right\}_{\alpha \in R}$ is orthonormal, we have $\left\|e_{\alpha}-e_{\beta}\right\|_{2}^{2}=\left\|e_{\alpha}\right\|_{2}^{2}+\left\|e_{\beta}\right\|_{2}^{2}=2$. Therefore,

$$
\begin{aligned}
& 2=\left\|e_{\alpha}-e_{\beta}\right\|_{2}^{2}=\varlimsup_{T \rightarrow \infty} \frac{1}{2 T} \int_{-T}^{T}\left|e^{i \alpha t}-e^{i \beta t}\right|^{2} d t \leq \\
& \leq \overline{\lim }_{T \rightarrow \infty} \frac{1}{2 T} \int_{-T}^{T}\left|e^{i \alpha t}-e^{i \beta t}\right|\left(\left|e^{i \alpha t}\right|+\left|e^{i \beta t}\right|\right) d t= \\
& =2 \varlimsup_{T \rightarrow \infty} \frac{1}{2 T} \int_{-T}^{T}\left|e^{i \alpha t}-e^{i \beta t}\right| d t=2\left\|e_{\alpha}-e_{\beta}\right\|_{1},
\end{aligned}
$$

i.e. $\left\|e_{\alpha}-e_{\beta}\right\|_{1} \geq 1$. Consequently, $V_{1}$ is non-separable. Now let's consider the space $V_{p}$ for $p>1$. Assume the contrary, i.e. assume that $V_{p}$ is separable and $M$ is a countable set everywhere dense in $V_{p}$. Then $\forall x \in V \exists x_{n} \in M$ : $\lim _{n \rightarrow \infty}\left\|x-x_{n}\right\|_{p}=0$. As $\forall x \in V$

$$
\|x\|_{1}=\varlimsup_{T \rightarrow \infty} \frac{1}{2 T} \int_{-T}^{T}|x(t)| d t \leq \varlimsup_{T \rightarrow \infty}\left(\frac{1}{2 T} \int_{-T}^{T}|x(t)|^{p} d t\right)^{\frac{1}{p}}=\|x\|_{p},
$$

we obtain $\lim _{n \rightarrow \infty}\left\|x-x_{n}\right\|_{1}=0$. Consequently, $M$ is a countable set everywhere dense in $V_{1}$, which contradicts the non-separability of $V_{1}$. So the space $V_{p}$ is non-separable.

Define a functional in $V_{p}$ by the following equality:

$$
e_{\alpha}^{*}(x)=\lim _{T \rightarrow \infty} \frac{1}{2 T} \int_{-T}^{T} x(t) e^{-i \alpha t} d t .
$$

The linearity of $e_{\alpha}^{*}$ is obvious. We have

$$
\left|e_{\alpha}^{*}(x)\right| \leq \varlimsup_{T \rightarrow \infty} \frac{1}{2 T} \int_{-T}^{T}|x(t)| d t \leq \varlimsup_{T \rightarrow \infty}\left(\frac{1}{2 T} \int_{-T}^{T}|x(t)|^{p} d t\right)^{\frac{1}{p}},
$$

i.e. $e_{\alpha}^{*} \in\left(V_{p}\right)^{*}$. Extending $e_{\alpha}^{*}$ by continuity onto $L_{p}^{V}(R)$, we obtain $e_{\alpha}^{*} \in$ $\left(L_{p}^{V}(R)\right)^{*}$. It is clear that $e_{\alpha}^{*}\left(e_{\beta}\right)=\delta_{\alpha \beta}$, i.e. the systems $\left\{e_{\alpha}\right\}_{\alpha \in R}$ and $\left\{e_{\alpha}^{*}\right\}_{\alpha \in R}$ are biorthogonal. 
Let $p \geq 2$ and $\forall x \in L_{p}^{V}(R)$. As $x \in L_{2}^{V}(R)$, it follows from Bessel's inequality that there are no more than a countable number of Fourier coefficients $e_{\alpha}^{*}(x)=\left(x, e_{\alpha}\right)_{V}$ that are different from zero and $\left\{e_{\alpha}^{*}(x)\right\}_{\alpha \in R} \in l_{2}(R)$. Further, from $l_{2}(R) \subset l_{p}(R)$ we obtain $\left\{e_{\alpha}^{*}(x)\right\}_{\alpha \in R} \in l_{p}(R)$. Consequently, for $p \geq 2$ the system $\left\{e_{\alpha}\right\}_{\alpha \in R}$ is $l_{p}(R)$-Besselian in $L_{p}^{V}(R)$.

The following theorem is true:

Theorem 3.1. Let $K$ be a $C B$-space with an uncountable unconditional basis $\left\{\delta_{\alpha}\right\}_{\alpha \in I}$. Then, in order for the pair $\left\{x_{\alpha} ; x_{\alpha}^{*}\right\}$ to be $K$-Bessel in $X$, it is necessary and, in case of completeness of $\left\{x_{\alpha}\right\}_{\alpha \in I}$ in $X$, sufficient that there exist an operator $T \in L(X, K)$ such that $T x_{\alpha}=\delta_{\alpha}, \forall \alpha \in I$.

Proof. Necessity. Let $\left\{x_{\alpha} ; x_{\alpha}^{*}\right\}$ be $K$-Bessel in $X$. Then $\forall x \in X\left\{x_{\alpha}^{*}(x)\right\}_{\alpha \in I} \in K$. Consider the operator $T: X \rightarrow K$ defined by the formula

$$
T(x)=\sum_{\alpha \in I} x_{\alpha}^{*}(x) \delta_{\alpha}
$$

It is clear that the equality $T x_{\alpha}=\delta_{\alpha}, \forall \alpha \in I$, holds. Define for every $\omega \in I^{a}$ the operator

$$
T_{\omega}(x)=\sum_{\alpha \in \omega} x_{\alpha}^{*}(x) \delta_{\alpha} .
$$

We have $T_{\omega} \in L(X, K)$ (see [5], [34]). Take an arbitrary $\forall x \in X$. Write elements of the set $I_{x}=\left\{\alpha: x_{\alpha}^{*}(x) \neq 0\right\}$ in the form of the sequence $\left\{\alpha_{n}\right\}_{n \in N^{*}}$. Put $\omega \cap I_{x}=$ $\left\{\alpha_{n_{k}}\right\}_{k \in N}$ for all $\omega \in I^{a}$. Then

$$
T_{\omega}(x)=\sum_{k=1}^{\infty} x_{\alpha_{n_{k}}}^{*}(x) \delta_{\alpha_{n_{k}}}
$$

So

$$
\sum_{k=1}^{\infty} x_{\alpha_{n_{k}}}^{*}(x) \delta_{\alpha_{n_{k}}}=\frac{1}{2}\left(\sum_{n=1}^{\infty} x_{\alpha_{n}}^{*}(x) \delta_{\alpha_{n}}+\sum_{n=1}^{\infty} \varepsilon_{n} x_{\alpha_{n}}^{*}(x) \delta_{n}\right),
$$

where $\varepsilon_{n}=1$ for $n=n_{k}$ and $\varepsilon_{n}=-1$ for $n \neq n_{k} . \forall x \in X$ we obtain that $\left\|T_{\omega}(x)\right\|_{K} \leq M_{x}<+\infty$. Then, by Banach-Steinhaus theorem, we have $\sup _{\omega \in I^{a}}\left\|T_{\omega}\right\|<+\infty$. As the boundedness of $T$ is equivalent to the condition $\omega \in I^{a}$ $\sup _{\omega \in I^{a}}\left\|T_{\omega}\right\|<+\infty$, the operator $T$ is bounded.

Sufficiency. Let the system $\left\{x_{\alpha}\right\}_{\alpha \in I}$ be complete in $X$ and there exist $T \in$ $L(X, K)$ such that $T x_{\alpha}=\delta_{\alpha}, \forall \alpha \in I$. Consider $\forall x \in X$. From $T x \in K$ it follows that $\left\{\delta_{\alpha}^{*}(T x)\right\}_{\alpha \in I} \in K$. We have

$$
\delta_{\alpha \beta}=\delta_{\alpha}^{*}\left(\delta_{\beta}\right)=\delta_{\alpha}^{*}\left(T x_{\beta}\right)=T^{*} \delta_{\alpha}^{*}\left(x_{\beta}\right), \forall \alpha, \beta \in I .
$$

Hence, due to the completeness of $\left\{x_{\alpha}\right\}_{\alpha \in I}$, we obtain

$$
T^{*} \delta_{\alpha}^{*}=x_{\alpha}^{*}
$$

Thus, taking into account (3.2), we obtain $\left\{x_{\alpha}^{*}(x)\right\}_{\alpha \in I}=\left\{\delta_{\alpha}^{*}(T x)\right\} \in K$. 
Corollary 3.1. Let $Y$ be a Banach space with an uncountable unconditional basis $\left\{\varphi_{\alpha}\right\}_{\alpha \in I}$ and $K_{\varphi}$ be a space generated by the system $\left\{\varphi_{\alpha}\right\}_{\alpha \in I}$. Then, in order for the pair $\left\{x_{\alpha} ; x_{\alpha}^{*}\right\}$ to be $K_{\varphi}$-Bessel in $X$, it is necessary and, in case of completeness of $\left\{x_{\alpha}\right\}_{\alpha \in I}$ in $X$, sufficient that there exist an operator $T \in L(X, Y)$ such that $T x_{\alpha}=\varphi_{\alpha}, \forall \alpha \in I$.

Corollary 3.2. Let the system $\left\{x_{\alpha}\right\}_{\alpha \in I}$ be complete in $X$ and $K$ be a $C B$ space with an uncountable unconditional basis $\left\{\delta_{\alpha}\right\}_{\alpha \in I}$. The system $\left\{x_{\alpha}\right\}_{\alpha \in I}$ is $K$-Bessel in $X$ only when there exists a number $M>0$ such that

$$
\left\|\left\{\lambda_{\alpha}\right\}\right\|_{K} \leq M\left\|\sum_{\alpha} \lambda_{\alpha} x_{\alpha}\right\|_{X}
$$

for every finite set of scalars $\left\{\lambda_{\alpha}\right\}$.

Proof. Let $\left\{x_{\alpha}\right\}_{\alpha \in I}$ be $K$-Bessel in $X$. Then, by Theorem 3.1, there exists a bounded operator $T \in L(X, K)$ defined by the formula (3.1). Let $\left\{\lambda_{\alpha}\right\}$ be an arbitrary finite set of scalars. Let $x=\sum_{\alpha} \lambda_{\alpha} x_{\alpha}$. Then $T x=\sum_{\alpha} \lambda_{\alpha} \delta_{\alpha}$ and

$$
\left\|\left\{\lambda_{\alpha}\right\}\right\|_{K}=\|T x\|_{K} \leq\|T\|\|x\|_{X}=\|T\|\left\|\sum_{\alpha} \lambda_{\alpha} x_{\alpha}\right\|_{X} .
$$

On the contrary, let there exist a number $M>0$ such that the inequality (3.3) holds for every finite set of scalars $\left\{\lambda_{\alpha}\right\}$. Consider arbitrary $\lambda=\left\{\lambda_{\alpha}\right\}_{\alpha \in I} \in K$. Define the operator $T: L\left(\left\{x_{\alpha}\right\}_{\alpha \in I}\right) \rightarrow K$ by the formula

$$
T\left(\sum_{\alpha} \lambda_{\alpha} x_{\alpha}\right)=\left\{\lambda_{\alpha}\right\} .
$$

Due to the minimality of the system $\left\{x_{\alpha}\right\}_{\alpha \in I}$, such an operator is defined correctly. By (3.3), the operator $T$ is bounded. Continuing the operator $T$ continuously to the whole $X$, we obtain the boundedness of $T$. As $T x_{\alpha}=\delta_{\alpha}, \forall \alpha \in I$, by Theorem 3.1, the system $\left\{x_{\alpha}\right\}_{\alpha \in I}$ is $K$-Bessel in $X$. Besselianness

Remark 3.1. Note that for $p \geq 2 l_{p}(R)$ - Besselianness of systems $e_{\alpha}(t)=e^{i \alpha t}$ in $L_{p}^{V}(R)$ immediately implies of Corollary 3.1. Indeed, for every finite set of scalars $\left\{\lambda_{\alpha}\right\}$ we have

$$
\left\|\left\{\lambda_{\alpha}\right\}\right\|_{l_{p}(R)} \leq\left\|\left\{\lambda_{\alpha}\right\}\right\|_{l_{2}(R)}=\left\|\sum_{\alpha} \lambda_{\alpha} e_{\alpha}\right\|_{2} \leq\left\|\sum_{\alpha} \lambda_{\alpha} e_{\alpha}\right\|_{p} .
$$

The next definition is a generalization of the concept of Hilbert sequence.

Definition 3.2. The pair $\left\{x_{\alpha} ; x_{\alpha}^{*}\right\}$ is called $K$-Hilbert in $X$ if the following condition holds: $\forall \lambda=\left\{\lambda_{\alpha}\right\}_{\alpha \in I} \in K \exists x \in X: \lambda=\left\{x_{\alpha}^{*}(x)\right\}_{\alpha \in I}$. If the system $\left\{x_{\alpha}\right\}_{\alpha \in I}$ is complete in $X$ and the pair $\left\{x_{\alpha} ; x_{\alpha}^{*}\right\}$ is $K$-Hilbert in $X$, then $\left\{x_{\alpha}\right\}_{\alpha \in I}$ is called $K$-Hilbert in $X$.

Example 3.2. The system $\left\{e_{\alpha}\right\}_{\alpha \in R}$ is $l_{p}(R)$-Hilbertian in $L_{p}^{V}(R)$ for $p \leq 2$. Indeed, consider $\forall \lambda=\left\{\lambda_{\alpha}\right\}_{\alpha \in R} \in l_{p}(R)$. Then $\lambda \in l_{2}(R)$. As $\left\{e_{\alpha}\right\}_{\alpha \in R}$ is an orthonormal system in $L_{2}^{V}(R)$, there exists $x \in L_{2}^{V}(R)$ such that $e_{\alpha}^{*}(x)=\left(x, e_{\alpha}\right)_{V}=$ 
$\lambda_{\alpha}$. From $L_{2}^{V}(R) \subset L_{p}^{V}(R)$ we obtain that $x \in L_{p}^{V}(R)$. Hence, $\left\{e_{\alpha}\right\}_{\alpha \in R}$ is $l_{p}(R)$ Hilbertian in $L_{p}^{V}(R)$ for $p \leq 2$.

The following theorem is true:

Theorem 3.2. Let $K$ be a $C B$-space with an uncountable unconditional basis $\left\{\delta_{\alpha}\right\}_{\alpha \in I}$. Then, in order for the pair $\left\{x_{\alpha} ; x_{\alpha}^{*}\right\}$ to be $K$-Hilbert in $X$, it is sufficient and, in case of completeness of $\left\{x_{\alpha}^{*}\right\}_{\alpha \in I}$ in $X^{*}$, necessary that there exist an operator $T \in L(K, X)$ such that $T \delta_{\alpha}=x_{\alpha}, \forall \alpha \in I$.

Proof. Necessity. Let $\left\{x_{\alpha} ; x_{\alpha}^{*}\right\}$ be $K$-Hilbert in $X$ and the system $\left\{x_{\alpha}^{*}\right\}_{\alpha \in I}$ be complete in $X^{*}$. Then $\forall \lambda=\left\{\lambda_{\alpha}\right\}_{\alpha \in I} \in K \exists x \in X$ such that $\lambda=\left\{x_{\alpha}^{*}(x)\right\}_{\alpha \in I}$. Due to the completeness of $\left\{x_{\alpha}^{*}\right\}_{\alpha \in I}$ in $X^{*}$, such an element is unique. Consider the operator $T: K \rightarrow X$ defined by the formula $T \lambda=x$. Obviously, $T \delta_{\alpha}=x_{\alpha}$, $\forall \alpha \in I$. It remains to show the boundedness of $T$. To this end, let's first prove its closedness. Let the sequence $\lambda_{n}=\left\{\lambda_{\alpha}^{(n)}\right\}_{\alpha \in I} \in K$ converge in $K$ to $\lambda=\left\{\lambda_{\alpha}\right\}_{\alpha \in I} \in K$ as $n \rightarrow \infty$, the sequence $T \lambda_{n}=x_{n}$ converge in $X$ to $y \in X$ as $n \rightarrow \infty$, and $T \lambda=x$. It is clear that $x_{\alpha}^{*}\left(x_{n}\right)$ converges to $x_{\alpha}^{*}(y)$ as $n \rightarrow \infty$. $\forall \alpha \in I$ we have

$$
\left|\lambda_{\alpha}^{(n)}-\lambda_{\alpha}\right|=\left|\delta_{\alpha}^{*}\left(\lambda_{n}-\lambda\right)\right| \leq\left\|\delta_{\alpha}^{*}\right\|\left\|\lambda_{n}-\lambda\right\|_{K} \rightarrow 0, n \rightarrow \infty .
$$

As $\lambda_{\alpha}^{(n)}=x_{\alpha}^{*}\left(x_{n}\right)$ and $\lambda_{\alpha}=x_{\alpha}^{*}(x)$, we have $x_{\alpha}^{*}(x)=x_{\alpha}^{*}(y)$. Hence, due to the completeness of $\left\{x_{\alpha}^{*}\right\}_{\alpha \in I}$, we obtain $x=y$. Consequently, $T \lambda_{n}$ converges in $X$ to $T \lambda$ as $n \rightarrow \infty$, i.e. the operator $T$ is closed. Then, by the closed graph theorem, $T$ is bounded.

Sufficiency. Let there exist an operator $T \in L(K, X)$ such that $T \delta_{\alpha}=x_{\alpha}$, $\forall \alpha \in I$. Consider arbitrary $\lambda=\left\{\lambda_{\alpha}\right\}_{\alpha \in I} \in K$. Let $T \lambda=x$. From $\lambda=\sum_{\alpha \in I} \lambda_{\alpha} \delta_{\alpha}$ we obtain

$$
x=T \lambda=\sum_{\alpha \in I} \lambda_{\alpha} T \delta_{\alpha}=\sum_{\alpha \in I} \lambda_{\alpha} x_{\alpha} .
$$

Therefore, $x_{\alpha}^{*}(x)=\lambda_{\alpha}$, i.e. $\lambda=\left\{x_{\alpha}^{*}(x)\right\}_{\alpha \in I}$.

Corollary 3.3. Let $Y$ be a Banach space with an uncountable unconditional basis $\left\{\varphi_{\alpha}\right\}_{\alpha \in I}$ and $K_{\varphi}$ be a space generated by the system $\left\{\varphi_{\alpha}\right\}_{\alpha \in I}$. Then, in order for the pair $\left\{x_{\alpha} ; x_{\alpha}^{*}\right\}$ to be $K_{\varphi}$-Hilbert in $X$, it is sufficient and, in case of completeness of $\left\{x_{\alpha}^{*}\right\}_{\alpha \in I}$ in $X^{*}$, necessary that there exist an operator $T \in$ $L(Y, X)$ such that $T \varphi_{\alpha}=x_{\alpha}, \forall \alpha \in I$.

Corollary 3.4. Let the system $\left\{x_{\alpha}\right\}_{\alpha \in I}$ be complete in $X$, the system $\left\{x_{\alpha}^{*}\right\}_{\alpha \in I}$ be complete in $X^{*}$, and $K$ be a $C B$-space with an uncountable unconditional basis $\left\{\delta_{\alpha}\right\}_{\alpha \in I}$. The system $\left\{x_{\alpha}\right\}_{\alpha \in I}$ is $K$-Hilbert in $X$ only when these exists a number $M>0$ such that

$$
\left\|\sum_{\alpha} \lambda_{\alpha} x_{\alpha}\right\|_{X} \leq M\left\|\left\{\lambda_{\alpha}\right\}\right\|_{K}
$$

for every finite set of scalars $\left\{\lambda_{\alpha}\right\}$.

Proof. Let $\left\{x_{\alpha}\right\}_{\alpha \in I}$ be $K$-Hilbert in $X$. By Theorem 3.2, there exists a bounded operator $T \in L(K, X)$ such that $T \delta_{\alpha}=x_{\alpha}, \forall \alpha \in I$. Then, for every finite set 
$\left\{\lambda_{\alpha}\right\}$ we have

$$
T\left\{\lambda_{\alpha}\right\}=T\left(\sum_{\alpha} \lambda_{\alpha} \delta_{\alpha}\right)=\sum_{\alpha} \lambda_{\alpha} x_{\alpha}
$$

Hence

$$
\left\|\sum_{\alpha} \lambda_{\alpha} x_{\alpha}\right\|_{X}=\left\|T\left\{\lambda_{\alpha}\right\}\right\| \leq\|T\|\left\|\left\{\lambda_{\alpha}\right\}\right\|_{K} .
$$

On the contrary, let the relation (3.4) hold for every finite set $\left\{\lambda_{\alpha}\right\}$. Define the operator $T$ for finite systems $\left\{\lambda_{\alpha}\right\}$ by the formula $T\left\{\lambda_{\alpha}\right\}=\sum_{\alpha} \lambda_{\alpha} x_{\alpha}$. From the inequality (3.4) it follows that this operator is bounded. Continuing it continuously to the whole $K$, we obtain the operator $T \in L(K, X)$. As $T \delta_{\alpha}=x_{\alpha}, \forall \alpha \in I$, from Theorem 3.2 it follows that the system $\left\{x_{\alpha}\right\}_{\alpha \in I}$ is $K$-Hilbert in $X$.

Remark 3.2. Note that for $p \leq 2 l_{p}(R)$ - Hilbertianness of systems $e_{\alpha}(t)=e^{i \alpha t}$ in $L_{p}^{V}(R)$ immediately implies of Corollary 3.2. Indeed, for every finite set of scalars $\left\{\lambda_{\alpha}\right\}$ we have

$$
\left\|\sum_{\alpha} \lambda_{\alpha} e_{\alpha}\right\|_{p} \leq\left\|\sum_{\alpha} \lambda_{\alpha} e_{\alpha}\right\|_{2}=\left\|\left\{\lambda_{\alpha}\right\}\right\|_{l_{2}(R)} \leq\left\|\left\{\lambda_{\alpha}\right\}\right\|_{l_{p}(R)} .
$$

\section{References}

[1] A. Aldroubi, Q. Sun, W.Sh. Tang, $p$-frames and shift invariant subspaces of $L_{p}, J$. Fourier Anal. Appl., 7(1) (2001), 1-22.

[2] N.K. Bari, Biorthogonal systems and bases in Hilbert space, Moscow Gos. Univ. Uceneye Zapiski, Matematika, 148(4) (1951), 69-107.

[3] B.T. Bilalov, Z.G. Guseinov, $p$-Bessel and $p$-Hilbert systems and $p$-bases, Dokl. NANA, LXIV(3) (2008), 3-8.

[4] B.T. Bilalov, Z.G. Guseinov, $K$-Bessel and $K$-Hilbert systems and $K$-bases, Doklady Mathematics, 80(3) (2009), 826-828.

[5] B.T. Bilalov, Some approximation problems, Baku, Elm, 2016.

[6] B.T. Bilalov, Sh.M. Hashimov, On Decomposition In Banach Spaces, Proceedings of the IMM of NAS of Azerbaijan, 40(2) (2014), 97-106.

[7] B.T. Bilalov, F.A. Guliyeva, Neotherian perturbation of frames. Pensee Jornal, 75:12, 2013, 425-431.

[8] B.T. Bilalov, F.A. Guliyeva, $t$-Frames and their Noetherian Perturbation, Complex Anal. Oper. Theory, 9(7) (2015), 1609-1631.

[9] B.T. Bilalov, Z.V. Mamedova, On the frame properties of some degenerate trigonometric system, Dokl. Nats. Akad. Nauk Azerb. LXVIII (5) (2012), 14-18

[10] B.T. Bilalov, F.A. Guliyeva, On The Frame Properties of Degenerate System of Sines, Journal of Function Spaces and Applications, Vol. 2012 (2012), Article ID 184186, 12 pages, doi:10. 1155/2012/184186.

[11] B.T. Bilalov, M.I.Ismailov, Y.I.Nasibov, Bessel families and uncountable frames in non-separable Hilbert spaces, Dokl. Nats. Akad. Nauk Azerb., 2017.

[12] B.T. Bilalov, M.I.Ismailov, Z.V.Mamedova, Bessel families and uncountable frames in non-separable Hilbert spaces, Azerbaijan Journal of Mathematics, 6(2), 2017.

[13] Ch. Chui, Wavelets: a tutorial in theory and applications, Academic Press, Boston, 1992.

[14] O. Christensen, An Introduction to Frames and Riesz Bases, Birkhäuser, Boston, 2002. 
[15] O. Christensen, Frames and bases. An introductory course, Birkhäuser, Boston, 2008.

[16] O. Christensen, D.T. Stoeva, $p$-frames in separable Banach spaces, Advances in Computational Mathematics 18(2003), 17-126.

[17] R. J. Duffin, A.C. Schaeffer, A class of nonharmonic Fourier series, Trans. Amer. Math. Soc. 72(1952), 341-366.

[18] I. Daubechies, Ten lectures on wavelets. SIAM, Philadelphia, 1992.

[19] H.G. Feichtinger, K.H., Gröchening, Banach spaces related to integrable group representations and their atomic decompositions I., J. Funct. Anal., 86(2) (1989), 307340 .

[20] H.G. Feichtinger, K.H. Gröchening, Banach spaces related to integrable group representations and their atomic decompositions II., Monatsh. Math. 108 (1989), 129-148.

[21] K.H. Gröchenig, Describing functions: atomic decomposition versus frames, Monatsh. Math., 112 (1991), 1 - 41.

[22] Ch. Heil, A Basis Theory Primer, Springer, 2011.

[23] S. Li, H., Ogawa Pseudoframes for subspaces with applications, J. Fourier Anal. Appl., 10 (2004), 409-431.

[24] S. Mallat, A wavelet tour of signal processing, Academic Press, San Diego, 1999.

[25] Y. Meyer, Wavelets and operators, Herman, Paris, 1990.

[26] A. Rahimi, B. Daraby, Z. Darvishi, Construction of Continuous Frames in Hilbert spaces, Azerbaijan Journal of Mathematics 7(1) (2017), 49-58.

[27] W. Sun, Stability of g-frames. Journal of Mathematical Analysis and Applications, 326 (2) (2007), 858-868.

[28] W. Sun, G-frames and g-Riesz bases. Journal of Mathematical Analysis and Applications, 322 (1) (2006), 437-452.

[29] S.R. Sadigova, On Frame Properties Of Degenerate System Of Exponents In Hardy Classes, Caspian Journal of Applied Mathematics, Ecology and Economics, 1(1) (2013), 97-103.

[30] S.R. Sadigova, I. Ismailov, On Frames of Double and Unary Systems in Lebesgue Spaces, Pensee Journal, 76(4) (2014), 189-202.

[31] S.R. Sadigova, Z.A. Kasumov, On atomic decomposition for Hardy classes with respect to degenerate exponential systems, Proc. of the IMM of NAS of Azerbaijan, 40(1) (2014), 55-67.

[32] S.R. Sadigova, On Frame Properties of Degenerate System of Exponents In Hardy Classes, Caspian Journal of Applied Mathematics Ecology and Economics, 1(1) (2013), 97-103.

[33] P.A. Terekhin, On Bessel systems in a Banach space, Matem. zametki, 91(2) (2012), 285-296.

[34] R. Young, An introduction to nonharmonic Fourier series, Academic Press, New York, 1980.

Migdad I. Ismailov

Baku State University, 23 Z. Khalilov str., Az.1148 Baku, Azerbaijan

Institute of Mathematics and Mechanics, National Academy of Sciences of Azerbaijan, 9 Vahabzadeh st., Az.1141, Baku, Azerbaijan

E-mail address: migdad-ismailov@rambler.ru

Received: November 12, 2018; Accepted: June 10, 2019 